\title{
Locally induced domain wall damping in a thin magnetic wire
}

\author{
G. Infante, ${ }^{1,2}$ R. Varga, ${ }^{1, a)}$ G. A. Badini-Confalonieri, ${ }^{2}$ and M. Vázquez ${ }^{2}$ \\ ${ }^{1}$ Institute of Physics, Faculty of Science, UPJS, 04154 Kosice, Slovakia \\ ${ }^{2}$ Instituto de Ciencia de Materiales de Madrid, CSIC, 28049 Madrid, Spain
}

(Received 11 May 2009; accepted 18 June 2009; published online 8 July 2009)

\begin{abstract}
The damping mechanisms affecting the motion of a single domain wall were studied in a thin bistable magnetic wire. It was found that the overall damping is frequency and temperature dependent through the locally induced anisotropy via structural relaxation. This phenomenon can increase the overall damping by one order of magnitude and enables an effective tailoring of the domain wall dynamics according to required application. (c) 2009 American Institute of Physics. [DOI: $10.1063 / 1.3174919$ ]
\end{abstract}

The controlled motion of domain walls in thin ferromagnetic wires is an important issue in magnetism. ${ }^{1-3}$ A general understanding of the dynamics of such boundaries in an imperfection containing material has been gained since the initial studies, but there are still aspects that remain under experimental investigation. Among them, energy dissipation mechanisms are of significant importance since damping governs the mobility of the domain wall and therefore controls its velocity.

The dynamic of a domain wall can be alternatively studied in amorphous glass-coated microwires ${ }^{4}$ due to their simple domain structure. They are casted by rapid solidification techniques and consist of a micron-sized soft ferromagnetic nucleus $(1-30 \mu \mathrm{m})$ covered by an insulating glass layer. The magnetic phenomena found in these wires includes bistable magnetic behavior for positive magnetostriction (Fe-based) compositions. Pronounced magnetoelastic and shape anisotropies determine for such alloy wires a "core-shell" domain structure. ${ }^{4,5}$ A large axially magnetized single domain with closing structures at the wire ends is surrounded by a radially oriented outer sheath multidomain structure. The resulting nearly ideal axial magnetization reversal process involves the depinning and propagation of a single $180^{\circ}$ domain wall from one closure structure when the switching field is reached. ${ }^{6}$ Therefore, the dynamics of a single domain wall can be investigated in these wires by means of simple, well-defined experiments that lead to interesting results such as very fast domain walls, ${ }^{7}$ sometimes supersonic $^{8}$ exhibiting supersonic boom, ${ }^{9}$ negative critical propagation field, or an additional source of damping. ${ }^{6,10}$

The aim of this paper is to present a detailed study of a domain wall damping mechanism based on structural relaxation. In some cases structural relaxation turns out to be the most important parameter affecting the domain wall dynamics and can increase damping up to one order of magnitude. It allows an efficient control of the magnetic viscosity by changing the locally induced anisotropy. Hence, the desired magnetic properties can be tailored according to technical requirements.

Positive magnetostriction glass-coated amorphous microwires with nominal composition $\mathrm{Fe}_{76} \mathrm{Si}_{9} \mathrm{~B}_{10} \mathrm{P}_{5}$ were produced by the quenching and drawing method. This alloy

\footnotetext{
a) Author to whom correspondence should be addressed. Electronic mail: rvarga@upjs.sk.
}

composition exhibits a combination of high glass forming ability, high saturation magnetization, and low coercive field. ${ }^{11}$ Pieces $10 \mathrm{~cm}$ in length with a $14 \mu \mathrm{m}$ thick ferromagnetic nucleus and a diameter about $40 \mu \mathrm{m}$ were selected for the experiments. The domain wall dynamics were studied by means of a Sixtus and Tonks-like setup ${ }^{12}$ composed of three coaxial coils: a magnetizing solenoid plus two symmetrically disposed pickup coils $60 \mathrm{~mm}$ apart. The damping was investigated varying the frequency of the applied magnetic field between $10 \mathrm{~Hz}$ and $2 \mathrm{kHz}$ and temperature dependent measurements were carried out in the range from $80 \mathrm{~K}$ up to $450 \mathrm{~K}$. More details can be found elsewhere. ${ }^{6-10}$

The domain wall dynamics are generally described by a linear dependence of the domain wall velocity $v$ on the applied magnetic field $H{ }^{13}$

$$
v=S\left(H-H_{0}\right),
$$

where $H_{0}$ is the critical propagation field and $S$ is the domain wall mobility $\left(S=2 \mu_{0} M_{s} / \beta\right.$, where $\mu_{0}$ is magnetic permeability of vacuum, $M_{s}$ is the saturation magnetization, and $\beta$ is the viscous domain wall damping).

Damping is the most important parameter defining the domain wall dynamics. Various causes of viscous damping $\beta$ have been identified and two of them are generally accepted: microeddy currents circulating in and near a moving domain wall are the more obvious cause of damping in metals. However, the eddy current parameter $\beta_{e}$ is negligible in large resistivity materials such amorphous microwires, ${ }^{14}$ which besides are very thin in diameter. The second well-known cause of energy dissipation is magnetic relaxation damping $\beta_{r}$ due to a delayed rotation of electron spins. This damping is related to the Gilbert damping parameter $\alpha^{15,16}$ and is inversely proportional to the domain wall width $\delta_{w}$,

$$
\beta_{r} \approx \alpha M_{s} / \gamma \delta_{w} \approx M_{s} \sqrt{K / A},
$$

where $\gamma$ is the gyromagnetic ratio, $A$ is the exchange stiffness constant, $K$ is the magnetic anisotropy energy (magnetoelastic one, $K=3 \lambda_{s} \sigma / 2$, in the case of amorphous microwires), $\lambda_{s}$ is the saturation magnetostriction, and $\sigma \propto\left(\alpha_{g}-\alpha_{m}\right) \Delta T$ are the mechanical stresses arising from the different thermal expansion coefficients of the glass cover $\alpha_{g}$ and of the metallic nucleus $\alpha_{m}$.

In the case of amorphous microwires, the abovementioned contributions cannot explain the temperature dependence of the overall damping. This fact is motivated in 


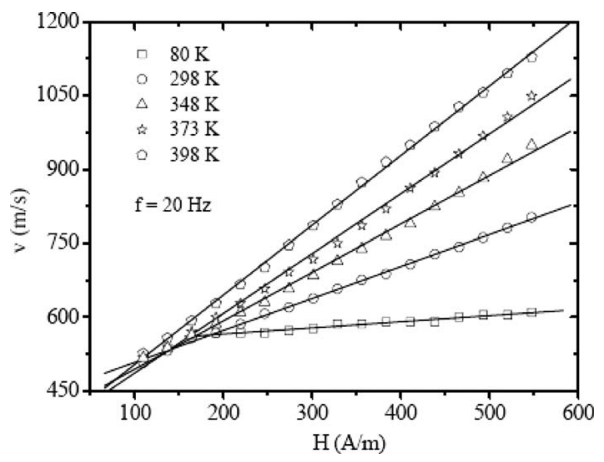

FIG. 1. Domain wall velocity as a function of applied field for the relaxed state $(20 \mathrm{~Hz})$. Temperature as a parameter. Full lines represent a linear fit.

the introduction of a third damping mechanism arising from structural relaxation. Structural relaxation processes are a consequence of the intrinsic metastable state of amorphous microwires. Hence, relaxation can occur even at low temperatures affecting most of its physical properties. ${ }^{17}$ These small displacements of mobile defects increase the local anisotropy are governed by a relaxation time and are strongly temperature dependent. Assuming that the thermal energy, $K T$, is higher than the splitting energy between two positions of the mobile defects, ${ }^{18}$ the damping term due to structural relaxation, $\beta_{s}$, is given by the following expression: ${ }^{6}$

$$
\beta_{s}(T, f) \propto \frac{\varepsilon_{p}^{2} c_{p}}{f_{r} K T}\left(1-e^{-f_{r} / 2 f}\right),
$$

where $c_{p}$ denotes the density of mobile defects, $\varepsilon_{p}$ corresponds to their interaction energy with the local spontaneous magnetization, $f$ is the frequency of the applied field, and $f_{r}=f_{0} e^{-Q / K T}$ is the relaxation frequency of the mobile defects; $f_{0}$ is a pre-exponential factor and $Q$ is their activation energy.

Figure 1 shows the expected linear domain wall dynamics, which were observed in a wide range of temperatures. The quantitative analysis of the experimental curves according to Eq. (1) provides information about the physical origin of the viscous damping. The resulting temperature dependence of the total damping $\beta$ is plotted in Fig. 2 for two magnetic field frequencies with the intention of showing the influence of structural relaxation. These frequencies, $20 \mathrm{~Hz}$ and $2 \mathrm{kHz}$, correspond to the sample been relaxed or unrelaxed, respectively. As previously mentioned, neither eddy

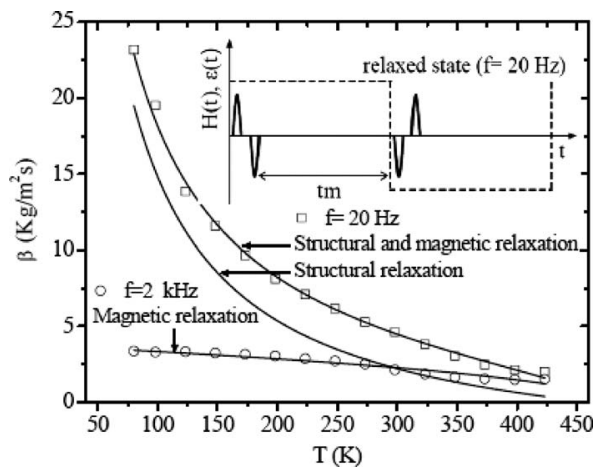

FIG. 2. Temperature dependence of the domain wall damping $\beta$ for the relaxed $(20 \mathrm{~Hz})$ and unrelaxed $(2 \mathrm{kHz})$ states. Full lines represent a fit according to corresponding contributions. Inset shows a schematic illustration for the relaxed state. Uniform drive field (dashed line) is shown as $H(t)$, induced voltage in sensing coils (full line) is depicted as $\varepsilon(t)$.

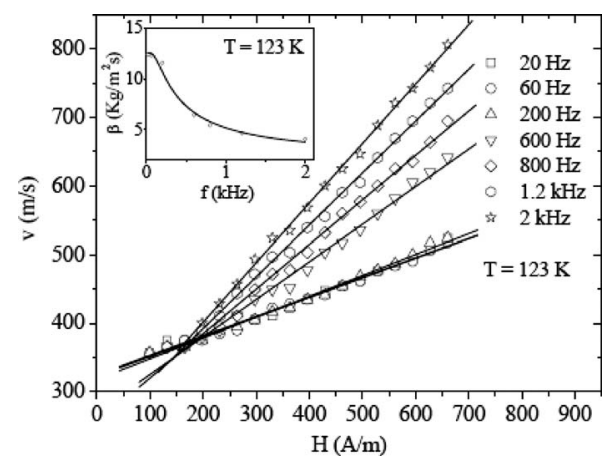

FIG. 3. Domain wall velocity as a function of applied field for $T=123 \mathrm{~K}$. Frequency as a parameter. Full lines represent a linear fit. Inset shows the corresponding frequency dependence of the domain wall damping $\beta$. Full line represent an exponential fit.

currents nor magnetic relaxation explain the observed temperature dependence of $\beta$ for a low measuring frequency (relaxed state, $20 \mathrm{~Hz}$ ): the small change in resistivity $(\Delta \rho$ $\approx 4 \%$ in the same temperature range) cannot be responsible for the order of magnitude change, and the temperature dependence of Eq. (2) does not explain the steep increase at low temperatures. It is the addition of magnetic and structural relaxation damping [Eqs. (2) and (3)], which accounts for the temperature dependence of $\beta$ in the relaxed state. As it can be noticed in Fig. 2, structural relaxation prevails at low temperatures but the presence of magnetic relaxation damping is also clearly noticeable. On the other hand, when the frequency of the applied field is increased (unrelaxed state, $2 \mathrm{kHz}$ ), the overall damping is much lower, especially in the low temperature regime, since magnetic relaxation damping turns out to be now the unique mechanism affecting the propagation of the domain wall.

The origin of the different behaviors found for the relaxed and unrelaxed states is schematically explained in the inset of Fig. 2. Structural relaxation is a time (frequency) dependent phenomena, so several characteristic time constants are involved: ${ }^{19}$ the intrinsic relaxation time of the mobile defects $\tau_{r}\left(=1 / f_{r}\right)$ and the available time for relaxation $t_{m}(\propto 1 / 2 f)$. The balance between them explains the global behavior. Due to the characteristics of the experimental setup, ${ }^{12}$ domain wall propagation (depicted as the two induced voltage pulses in the inset of Fig. 2) is observed almost immediately after the change of the external magnetic field. When the domain wall passes, it redistributes the mobile defects and consequently leaves random local anisotropy. After domain wall propagation, the applied field still remains constant for a certain time $t_{m}$ during which the domain structure is able to stabilize before the applied field is inverted again and the domain wall propagates in opposite direction repeating the measuring cycle.

For a low measuring frequency (relaxed state; inset of Fig. 2), $t_{m}>\tau_{r}$. The domain structure relaxes increasing the locally induced anisotropy. In fact, the frequency dependent domain wall dynamics presented in Fig. 3 show a low mobility in this case. On the contrary, when the frequency of the applied field is high enough (unrelaxed state), $t_{m}<\tau_{r}$. The domain structure has no time to relax, therefore the sample remains with very small locally induced anisotropy and the mobility increases (see Fig. 3).

Such a relaxation effect is confirmed by the frequency dependence of the domain wall damping $\beta$ shown in the 
inset of Fig. 3. It follows the expected exponential decay function for a structural relaxation contribution in accordance to Eq. (3). Small deviations should be ascribed to the assumption of a single relaxation time; while the relaxation behavior is generally interpreted in terms of a distribution of relaxation times. ${ }^{17}$

As a conclusion, it has been shown that structural relaxation damping is an effective way to manipulate the domain wall dynamics since it can control damping within one order of magnitude by changing the local anisotropy. The effect of structural relaxation decreases with temperature and frequency, reflecting in the latter case the ratio between the measuring frequency and the relaxation frequency of the mobile defects. Hence, it can be enhanced (at low frequencies) or removed (at higher frequencies). This mechanism of domain wall damping can either be induced reversibly (the case presented here) or irreversibly (e.g., by magnetic or stress annealing) even in crystalline materials, ${ }^{13}$ widely employed in applications based on nanowires. ${ }^{1-3}$

The authors thank Professor J. I. Íñiguez for valuable discussion. This work was supported by the Spanish Ministry of Science and Innovation (MICINN) Project No. MAT200765420-C02-01, as well as by the Slovak Ministry of Education Grant Nos. VEGA-1/0076/09 and MVTS 6RP/Manunet/ UPJS/08. G. Infante acknowledges Spanish MICINN FPU fellowships program.
${ }^{1}$ G. S. D. Beach, C. Nistor, C. Knutson, M. Tsoi, and J. L. Erskine, Nature Mater. 4, 741 (2005).

${ }^{2}$ D. A. Allwood, G. Xiong, C. C. Faulkner, D. Atkinson, D. Petit, and R. P. Cowburn, Science 309, 1688 (2005).

${ }^{3}$ S. S. P. Parkin, M. Hayashi, and L. Thomas, Science 320, 190 (2008).

${ }^{4}$ M. Vázquez, Handbook of Magnetism and Advanced Magnetic Materials (Wiley, Chichester, West Sussex, England, 2007), Vol. 4, p. 2193.

${ }^{5}$ H. Chiriac and T. A. Ovari, Prog. Mater. Sci. 40, 333 (1996).

${ }^{6}$ R. Varga, K. L. Garcia, M. Vázquez, and P. Vojtaník, Phys. Rev. Lett. 94, 017201 (2005).

${ }^{7}$ R. Varga, A. Zhukov, J. M. Blanco, M. Ipatov, V. Zhukova, J. Gonzalez, and P. Vojtaník, Phys. Rev. B 74, 212405 (2006).

${ }^{8}$ R. Varga, A. Zhukov, V. Zhukova, J. M. Blanco, and J. Gonzalez, Phys. Rev. B 76, 132406 (2007).

${ }^{9}$ R. Varga, K. Richter, A. Zhukov, and V. Larin, IEEE Trans. Magn. 44, 3925 (2008)

${ }^{10}$ R. L. Novak, J. P. Sinnecker, and H. Chiriac, J. Phys. D: Appl. Phys. 41, 095005 (2008).

${ }^{11}$ A. Makino, T. Kubota, C. Chang, M. Makabe, and A. Inoue, J. Magn. Magn. Mater. 320, 2499 (2008).

${ }^{12}$ K. J. Sixtus and L. Tonks, Phys. Rev. 37, 930 (1931).

${ }^{13}$ B. D. Cullity, Introduction to Magnetic Materials (Addison-Wesley, Menlo Park, 1972), p. 446.

${ }^{14}$ R. P. del Real, C. Prados, D. X. Chen, A. Hernando, and M. Vázquez, Appl. Phys. Lett. 63, 3518 (1993).

${ }^{15}$ T. H. O'Dell, Ferromagnetodynamics (MacMillan, London, 1981), p. 46.

${ }^{16}$ R. C. O'Handley, J. Appl. Phys. 46, 4996 (1975).

${ }^{17}$ H. Kronmüller and M. Fähnle, Micromagnetism and the Microstructure of Ferromagnetic Solids (Cambridge University Press, Cambridge, 2003), p. 274.

${ }^{18}$ H. Kronmüller, Phys. Status Solidi B 118, 661 (1983).

${ }^{19}$ L. M. García, J. Bartolomé, F. J. Lázaro, C. de Francisco, and J. M. Muñoz, Phys. Rev. B 54, 15238 (1996). 